\title{
Vers des relations durables entre insectes auxiliaires, cultures et protection intégrée
}

Jacqueline PIERRE

INRA UMR 1099 BiO3P (Biologie des populations

appliquée à la protection des plantes),

Domaine de la Motte, BP 35327,

F 35653 Le Rheu

$<$ <acqueline.pierre@rennes.inra.fr>

\begin{abstract}
One purpose of the sustainable agriculture is not having adverse effects on the beneficial insects. Presently non target insects are still exposed to high doses of various pesticides used on various crops. The major beneficial insects are domesticated pollinators such as honeybee. Regarding this one, no direct correlation between diffuse mortality, sublethal effects or colony collapse disorder and pesticides is clearly demonstrated. Nevertheless, studies have shown the complexity of the combined toxic effects between pesticides (principally synergy between insecticides and fungicides), their incidence on the insect health and their putative relationship with insect pathogens (bacteria, virus, fungi or mites). That leads to consider that the reduction of pesticides (qualitatively and quantitatively) is needed to prevent non target insects from excessive mortality even if other causes of over mortality exist.
\end{abstract}

Key words: sustainable agriculture, beneficial insects, toxic effects between, pesticide

\section{Qui sont les insectes auxiliaires que I'on souhaite protéger et comment?}

Parmi les insectes auxiliaires figurent les insectes prédateurs ou parasites $d^{\prime}$ autres insectes qui sont, eux, nuisibles. C'est le cas notamment, chez les brassicaceae, de l'hyménoptère parasitoïde Trybliographa rapae ou du staphyllin Aleochara bilineata, parasites des larves de la mouche du chou Delia radicum, de diaeretiella rapae parasitoïde du puceron cendré Brevicoryne brassicae. Cependant, les insectes auxiliaires qui viennent le plus spontanément à l'esprit sont les pollinisateurs, plus particulièrement certains hyménoptères sociaux (vivant en colonie) ou non. Parmi ceux-ci, I'abeille domestique (Apis mellifera) tient la toute première place non seulement en raison de son rôle économique mais aussi de son abondance relative. Les bourdons sont aussi des hyménoptères sociaux qui jouent un rôle notable dans la pollinisation et certaines espèces font actuellement l'objet d'élevage tel le Bombus terrestris en Europe. En Amérique du Sud, on commence à prendre très au sérieux l'élevage et l'utilisation d'abeilles mélipones (dites « sans dard ») pour la pollinisation. Certaines espèces d'hyménoptères non sociaux sont également élevées en grand nombre et utilisées efficacement en Amérique du Nord principalement.

L'abeille domestique étant I'hyménoptère pollinisateur dont la biologie est la mieux connue, nous nous focaliserons surtout sur cette espèce à titre de modèle.
D’autre part, protéger les insectes auxiliaires, ennemis naturels ou pollinisateurs, passe par des conduites culturales permettant à leurs populations de se développer correctement voire d'être introduites et maintenues au champ ou en milieu fermé. Parmi ces techniques, la réduction des pesticides joue un rôle majeur, c'est aussi pour cette raison que nous nous focaliserons sur la toxicité des pesticides.

\section{Comment déterminer la toxicité des produits phytosanitaires?}

Avant leur mise sur le marché, les produits phytosanitaires sont soumis à un examen de leur toxicité vis-à-vis des insectes auxiliaires et des pollinisateurs, examen s'inscrivant dans le cadre du processus de mise sur le marché (encadré 1 et [1]).

Certains des essais demandés au plan communautaire sont connus en France sous les démoninations «méthode CEB 95 » et «méthode CEB 230 » (ex-129).

La méthode 95 est l'équivalent de l'essai de toxicité aiguë sur abeille adulte proposé par I'OCDE. Elle est effectuée sur des abeilles en laboratoire et a pour but de définir la toxicité intrinsèque de la matière active du produit voire de ses dérivés métaboliques. C'est un test de toxicité aiguë (application par voie orale ou topique) permettant de déterminer la dose de produit conduisant, après une seule application, à la mortalité de $50 \%$ des individus testés (DL50).
La méthode 230, également un équivalent de méthodes internationales, est pratiquée sous tunnel et concerne les abeilles butineuses; dans ce cas, le produit est appliqué sur la plante et son effet toxique est évalué en comptabilisant les abeilles mortes au sol avant et après application.

Ces tests portent donc sur un effet létal à court ou moyen terme chez l'abeille adulte en milieu non naturel. Dans la réalité, il existe plusieurs scénarios possibles allant de l'effet létal immédiat par application unique d'une forte dose (toxicité aiguë) jusqu'à un effet sublétal ne se révélant que très tardivement suite à une exposition chronique à de faibles doses. La notion de niveau d'exposition est d'ailleurs déterminante car dans les faits c'est bien la ou les doses auxquelles seront exposées les abeilles, une ou plusieurs fois, qui auront éventuellement un effet toxique.

Par ailleurs, il est nécessaire de prendre en compte le fait que l'abeille est un insecte social dont les colonies sont de très grande taille (jusqu'à 50000 individus). Cette spécificité implique d'une part que les effets cumulatifs ne portent pas uniquement sur la butineuse considérée individuellement mais sur l'ensemble des butineuses qui rapportentà la ruche du pollen et du nectar (ce dernier donnant le miel). De plus, ces stocks vont constituer la nourriture des larves ou des abeilles « d'intérieur » qui ne sont pas encore devenues des butineuses. Ceci va avoir une incidence sur le développement de la colonie. Il semble par conséquent logique que, pour connaître le réel impact des matières toxiques sur la colonie, il faille être en mesure de le tester 


\section{Encadré 1 \\ Les requis de la réglementation sur les produits phytopharmaceutiques}

Auteur : Anne Alix. Agence française de sécurité sanitaire des aliments, Direction du végétal et de l'environnement, 10 rue Pierre Curie, 94704 Maisons Alfort Cedex

Dans le cas des auxiliaires et des pollinisateurs, la relation aux cultures est importante, aussi font-ils l'objet d'une évaluation des risques dédiée, dans le cadre des évaluations préalables à la mise sur le marché des produits phytopharmaceutiques. L'objectif de cette évaluation est d'estimer la toxicité intrinsèque des substances actives et produits commerciaux pour la faune auxiliaire non cible, et d'apprécier qualitativement et quantitativement les risques éventuels liés à une exposition de cette faune aux résidus (incluant la substance active et ses produits de dégradation) du produit commercial utilisé sur la culture. Cette évaluation est un requis réglementaire, préalable à toute mise sur le marché des produits phytopharmaceutiques et ce depuis 1996, date d'entrée en vigueur de la Directive 91/414/EC et de ses directives filles dans les états de I'Union européenne. Elle est harmonisée au plan communautaire et conduite de façon systématique, pour chaque usage des produits commerciaux. En France, c'est la Direction du végétal et de l'environnement de l'AFSSA qui assure ces évaluations.

La toxicité des produits phytopharmaceutiques pour les auxiliaires est estimée dans un premier temps au moyen d'essais en laboratoire, réalisés sur deux espèces modèles, l'hyménoptère parasitoïde Aphidius rhopalosiphi, et l'acarien prédateur Typhlodromus pyri. Ces deux espèces ont été sélectionnées pour leur sensibilité importante aux produits phytopharmaceutiques, mise en évidence par l'analyse d'une base de données Dans le cas où la toxicité intrinsèque ainsi estimée conduit à identifier un risque potentiel au champ, ces deux essais sont complétés par une batterie d'essais impliquant d'autres espèces prédatrices et parasitoïdes selon des protocoles expérimentaux de complexité croissante selon les besoins. L'objet de cette batterie d'essais est de se placer dans des conditions d'exposition des arthropodes testés reproduisant au plus près la réalité et les conditions d'emploi prévues du produit. La multiplication des espèces modèles permet de réduire l'incertitude liée à des différences de sensibilités inter espèces. L'échelle ultime d'investigation est l'étude en champ. Toutes les substances destinées à un emploi en plein champ ont fait l'objet d'essais sur les deux espèces modèles et $60 \%$ d'entre elles ont fait l'objet d'une évaluation approfondie au moyen d'essais additionnels.

La toxicité des produits phytopharmaceutiques pour les espèces pollinisatrices est évaluée en prenant l'abeille domestique, Apis mellifera, comme espèce modèle. Comme dans le cas des auxiliaires, toutes les substances destinées à un emploi en plein champ font l'objet d'essais de toxicité aiguë exposant des adultes à la substance ou au produit commercial par voie orale et par contact. Lorsqu'un risque possible est identifié pour un usage donné, des essais supplémentaires sont requis, lesquels visent à s'assurer que l'usage en question n'entraîne pas d'effet sur « les larves d'abeilles, sur le comportement des abeilles et sur la survie et le développement ». Ces essais se déclinent en cage, sous tunnel et au champ, et mettent en œuvre, selon le profil de la substance et le degré de réalisme souhaité pour l'évaluation, des cultures traitées avec le produit, l'exposition via des nourrisseurs et des colonies de taille adaptée aux surfaces expérimentales. Pour les produits destinés au traitement du sol ou de semences et présentant des propriétés de systémie entrainant la présence de résidus dans les parties florales, et plus particulièrement le pollen et/ou le nectar, une approche dédiée a été développée par un groupe de travail de I'ICPBR (International Commission on Plant-Bee Relationships), piloté par I'AFSSA. Cette approche inclut de nouveaux protocoles d'essais adaptés aux produits utilisée en traitement de sol et de semence, prenant en compte l'exposition des butineuses sur la durée de la floraison (essai de toxicité sur adultes durant 10 jours) ou permettant les investigations sur couvain sous tunnel (test tunnel OCDE). Un essai de toxicité en laboratoire sur larves d'abeille a par ailleurs été mis au point, lequel offrira, une fois totalement validé, les possibilités d'un screening des propriétés écotoxiques qui inclue les stades de développement.

Enfin, les essais en champ sont adaptés aux traitements de sol et semence, afin de prendre en compte les aspects liés à la persistance et à la mortalité hivernale.

sur les différents individus la constituant, voire sur la survie globale de la colonie.

Force est de constater que par le passé les tests ont été trop rudimentaires pour évaluer correctement la toxicité des produits. « L'affaire Gaucho ", dès 1993, a au moins permis de mettre en exergue les difficultés à lever : limite de capacité de détection de la dose d'exposition dans le pollen et le nectar dans le cas des produits systémiques, toxicité des métabolites de la matière active après ingestion par l'abeille, durée de rémanence ou mode de dégradation du produit. Depuis, la Direction du végétal et de l'environnement, de l'Agence française de sécurité sanitaire et alimentaire (AFSSA), travaille à l'amélioration des tests et des méthodes d'évaluation des risques (encadré 1). En France, des propositions ont été faites concernant la méthode 230. II s'agirait de suivre un protocole expérimental permettant de prendre en compte la mortalité du couvain, les pontes, l'intensité de butinage à des nourrisseurs placés dans le tunnel, la prise de poids des colonies [2]. En ce qui concerne l'effet toxique sur les larves, une méthode d'élevage de larves a été mise au point à I'INRA afin de réaliser des tests standardisés pouvant être intégrés aux processus d'évaluation communautaires [3]. Des essais au champ font également partie intégrante des nouveaux schémas.

Malgré les améliorations apportées à l'évaluation d'un produit, qui risquent d'ailleurs de conduire à un durcissement de la procédure $d^{\prime}$ homologation, les spécialistes en toxicologie soulignent d'autres difficultés propres à toute étude des toxiques et elles sont nombreuses. Ainsi, l'effet d'un toxique n'est pas forcément directement dépendant de la dose appliquée : selon leur mode d'action, certaines molécules peuvent se révéler plus nocives à faible dose qu'à forte dose ou bien présenter deux doses létales différentes. La toxicité peut dépendre non seulement de la période de la vie de l'animal mais aussi des conditions environnementa- les [4] (température ; application sur tel ou tel support végétal). Il existe des interactions entre les différents produits et celles-ci peuvent être soit un simple effet cumulatif, un effet inhibiteur ou au contraire un effet synergique. C'est ce dernier cas qui est le plus alarmant. Ainsi, il a été démontré en laboratoire que l'association, à des doses sublétales, entre deux insecticides neurotoxiques (carbamate insecticide et pyréthrinoïde) ou entre un insecticide et un fongicide (pyréthrinoïde et triazol ou imidazole) [5] devenait mortelle alors que chacun des produits avait peu voire pas d'effet. Outre ces difficultés, il est évident que les effets sublétaux susceptibles de se déclarer sur le long terme sont difficiles à détecter pour différentes raisons : le lien de cause à effet peut passer inaperçu, les symptômes peuvent être ténus ou confondus avec des maladies infectieuses ou encore hors de portée d'observation directe. Les symptômes peuvent consister en des difficultés de la butineuse à voler correctement, à s'orienter, à 
communiquer le lieu de la ressource [6], à apprécier correctement la valeur nutritive de la ressource (modification de la sensibilité au sucre [7]), à apprendre correctement à reconnaître une odeur $[8,9]$ ou consister en des troubles métaboliques des réserves lipidiques et glucidiques de l'abeille. Plus insidieusement, une accumulation progressive d'un ou plusieurs produit(s) dans la cire, maintes fois triturée par les abeilles, peut jouer un rôle différé.

Reste à déterminer dans quelle mesure ces observations faites en conditions contrôlée surviennent sur le terrain et quel rôle elles jouent dans l'évolution de l'état sanitaire global des pollinisateurs.

\section{Quel est le rôle des pesticides dans le « déclin des abeilles " ?}

Les media se font actuellement abondamment l'écho du « déclin des abeilles ». Le mot déclin recouvre deux phénomènes qui semblent distincts. D'une part la surmortalité, d'autre part le syndrome de disparition des abeilles décrit aux Etats-Unis sous le terme de CCD (Colony Collapse Disorder). Dans le premier cas, il s'agit d'une mortalité excessive ou d'un fort affaiblissement des colonies qui touche les abeilles à certaines périodes de la vie de la colonie, tout particulièrement au sortir de I'hiver. Ce phénomène est surtout décrit actuellement en Europe et la France est touchée. Les premières enquêtes sérieuses conduites par le CNDA (Centre national de développement apicole) montrent qu'en France le taux de perte hivernale de colonies avoisine les $30 \%$ avec de fortes variations selon les régions, le Grand Est étant le plus atteint [10]. Cette surmortalité se traduit non seulement par la présence d'abeilles mourantes ou mortes mais aussi par une diminution des réserves, une diminution du couvain. Dans le cas du CCD, les symptômes sont différents : les ouvrières disparaissent de la ruche, les réserves sont correctes et le couvain sain, ceci donnant l'impression que la colonie a été récemment désertée. Ce syndrome a déjà touché 35 États des États-Unis et semble s'étendre à une partie du Canada. Selon les ruchers, les pertes peuvent être très sévères. II est bien évidemment difficile de dire si dans tous les cas déclarés la distinction a été correctement faite entre les deux phénomènes et il semblerait que des cas de CCD existent en Europe tandis que les États-Unis connaissent également des cas de surmortalité.

Des recherches sont en cours afin de déterminer les causes exactes de ces phénomènes. Les hypothèses mises en avant sont multiples et bien souvent communes aux deux situations. Pour le CCD, on évoque [11] spécifique- ment l'apparition ou l'extension de nouvelle(s) maladie(s) virale(s) ou d'une nouvelle espèce de varroa (l'acarien des trachées), mais en général les pertes de colonies sont imputées à un affaiblissement de celles-ci en raison de la présence du varroa "classique ", varroa destructor. Cet acarien joue le rôle d'un vrai Dracula en pompant I'hémolymphe des larves et des adultes. Sont également mis en cause : le surmenage des colonies par des transhumances répétées et sur de longues distances, le manque de nourriture diversifiée et adaptée. Les cultures OGM sont également soupçonnées par certains alors que tous les travaux de recherche ont montré leur innocuité [12]. Plus fondamentalement, certains chercheurs considèrent que la diversité génétique des abeilles s'est restreinte au cours de sélections mal dirigées et que les capacités naturelles des abeilles à lutter, par des comportements adaptés, contre certaines attaques (en particulier le varroa) auraient largement disparu.

Quoi qu'il en soit de la détermination de la cause majeure de la perte des colonies, la plupart de ces investigations prennent en compte des causes multifactorielles et s'accordent pour considérer que les pesticides sont un des éléments possibles de l'affaiblissement des abeilles. Cependant, sur ce sujet, diverses opinions s'affrontent, les uns mettant en avant les pesticides tandis que d'autres considèrent qu'ils ne sont qu'un facteur aggravant.

Des travaux récents [13] de l'AFSSA ont montré qu'il n'y a pas de lien statistique entre l'état de santé de la colonie (mesuré par le taux de mortalité, le nombre d'individus adultes sains et l'abondance du couvain) et les résidus de pesticides (mesurés dans diverses matrices telles que le corps des abeilles, le miel, le pollen, la cire). Cette étude a porté sur 120 colonies provenant de 24 ruchers différents répartis dans 5 régions de France fortes productrice de miel, dont du miel de colza. Elle montre également que les résidus de pesticides sont présents à faibles doses dans toutes les matrices, ces doses étant similaires à ce qui a été détecté par d'autres chercheurs en Europe ou aux ÉtatsUnis il y a plusieurs années. La fréquence relative de chaque pesticide n'est pas équivalente selon la matrice considérée, ni même selon la saison. Cependant, l'imidaclopride (matière active de l'insecticide Gaucho) et un de ses métabolites sont le plus fréquemment retrouvés aussi bien dans les pelotes de pollen que dans le miel ou le corps des abeilles. Parmi ces pesticides il faut signaler la présence, dans la cire, d'acaricides (Tau fluvalinate et coumaphos) utilisés par les apiculteurs eux-mêmes pour lutter contre le varroa destructor. Les auteurs de cette publication, tout en montrant l'absence de lien direct entre pesticides et mortalité, font preuve de prudence en concluant que des travaux complémentaires sont nécessaires afin d'étudier les effets synergiques entre les résidus mais également leurs interactions possibles avec les divers agents pathogènes (bactérie, virus, varroa).

Pour résumer, les pertes de colonies auxquelles on assiste actuellement sont sans doute la résultante de causes multiples dont il faut détecter l'élément déterminant afin de mettre en place une lutte ciblée. Cet élément déterminant peut être différent selon que l'on veut lutter contre le CCD ou une surmortalité. Par contre, l'action des pesticides est sans aucun doute un facteur favorisant dans tous les cas. Plutôt que d'incriminer uniquement les pesticides comme certains sont tentés de le faire et d'abandonner ainsi trop facilement d'autres pistes, l'attitude la plus efficace consiste à mener le combat sur plusieurs fronts, réduction des pesticides incluse.

\section{Quelles seraient les incidences agronomiques d'un déclin des pollinisateurs ?}

On prête (vraisemblablement à tort) la phrase suivante à Einstein : "Si les abeilles venaient à disparaître, I'humanité n'aurait plus que 4 ans à vivre ». Qu'en est-il exactement?

Cette assertion suppose que I'on connaisse le niveau de dépendance de chaque plante cultivée vis-à-vis de la pollinisation par les insectes et surtout l'abeille que l'on est en mesure de gérer du fait de son caractère domestique. Des estimations ont été faites dans ce sens mais il faut bien reconnaître que le niveau de dépendance est rarement déterminé de manière fiable $[14$, 15], d'autant plus que la biologie florale initiale de la plante est sujette à manipulation de la part des sélectionneurs. Ainsi une plante originellement plus ou moins autogame et pouvant se passer des insectes est transformée, par les sélectionneurs, en allogame de manière à obtenir des hybrides plus performants. Dans ce cas, la plante a besoin des insectes pour que les transferts de pollen soient assurés. Le colza en est une illustration. Initialement, les variétés de colza classique sont hermaphrodites et considérées comme largement autogames et par conséquent comme pouvant se passer des insectes. En réalité, cette première idée est fausse car divers travaux, anciens ou très récents, montrent que même dans un tel cas les butineurs permettent d'améliorer le rendement $[16,17]$. Depuis, des colzas hybrides ou des composites hybrides lignée ont été conçus pour bénéficier de la vigueur hybride. Dans ce cas, que ce soit pour la production de semences hybrides ou pour la production agricole utilisant les composites hybrides lignée, la présence 
de pollinisateurs devient indispensable pour maximiser le transfert de pollen entre les parents mâles et les femelles (figure 1). Cette dépendance est quasi-totale sachant que la part de pollinisation par le vent chez le colza est faible [17]. On voit donc bien ici que le degré de dépendance vis-à-vis de la pollinisation entomophile est parfois difficile à établir.

En revanche, il existe des cas où la dépendance quand elle est forte et manifeste ne peut être mise en doute. Une étude effectuée par la FAO (Food and Agriculture Organization ${ }^{1}$ ) en 2004 portant sur 107 plantes cultivées présentant un fort intérêt économique montre que 13 espèces végétales dépendent très fortement des pollinisateurs et 30 en dépendent de manière significative. Malgré les restrictions que nous avons pu émettre précédemment, on peut donc estimer de manière assez fiable qu'environ $40 \%$ (43/107) des plantes nécessaires à la consommation humaine et produites en quantité importante (plus de $4 \times 10^{6}$ tonnes/an/ espèce) dépendent des insectes.

Notre vulnérabilité vis-à-vis de ce problème de dépendance alimentaire et économique [14, 15] (estimée à $153.10^{9}$ euros/an au niveau mondial) a dernièrement conduit les décideurs à favoriser la mise en place de programmes portant à la fois sur la recherche des causes du CCD (le United States Department of Agriculture a alloué $66.10^{6}$ euros $/ 5$ ans), de la surmortalité des abeilles (programme suisse Agroscope) voire des deux (programme international "Coloss »). D'autres programmes à caractère plus écologique prennent en compte la protection et la bioconservation des insectes pollinisateurs en général (projet européen «Alarm », module Pollinator loss; projet mondial « International Pollinator Initiative » créé en 2000). En effet, parmi ces insectes figurent une multitude d'espèces d'hyménopères que nous avons évoqués succinctement en introduction. Ceux-ci fournissent des "services gratuits » pour la pollinisation [18] et il est important de les protéger tant pour des raisons agronomiques qu'écologiques.

La France participe aux projets internationaux et poursuit plus modestement une réflexion sur la mise en place de jachères fleuries pour les pollinisateurs afin de corriger le manque de diversité et le côté sporadique des ressources alimentaires des abeilles du fait des monocultures [19]. Ceci ne va d'ailleurs pas sans poser des problèmes législatifs malgré la simplicité apparente de cette dernière proposition. Toujours en France, il est actuellement question, suite au rapport Saddier [20] en 2008, de créer un

${ }^{1}$ http://faostat.fao.org

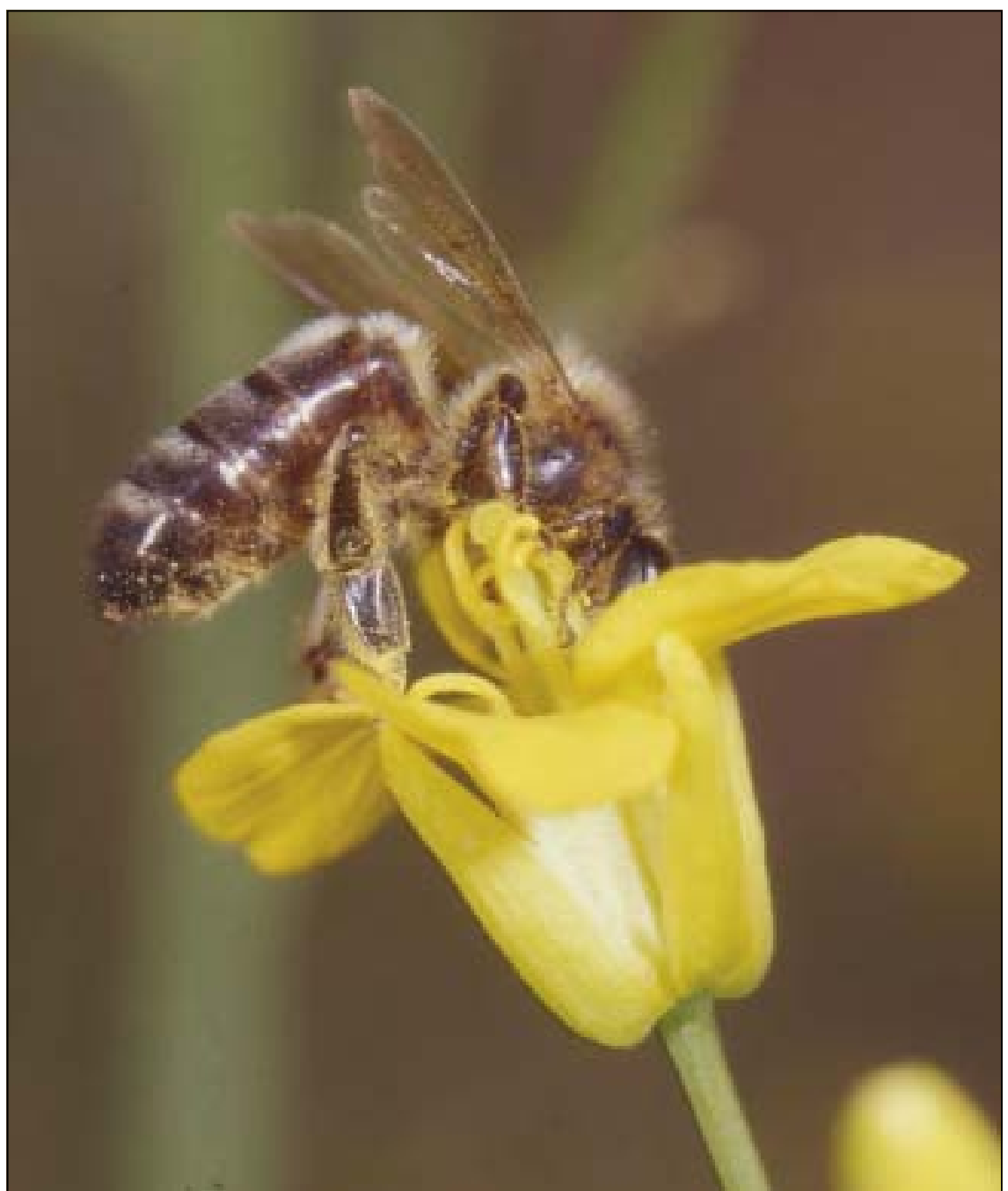

Figure 1. Abeille butineuse de nectar sur colza (photo : INRA)

Institut scientifique et technique apicole (ISTA) et de favoriser l'enseignement de l'apiculture par la création d'un BTS.

\section{Quel est l'intérêt d'une approche intégrée pour la sauvegarde des insectes auxiliaires?}

La lutte intégrée suppose de diversifier et cumuler les moyens de lutte : culturale, génétique et chimique.

Sur ce dernier point, un des objectif affichés du Grenelle de l'environnement est de réduire les intrants phytosanitaires de $50 \%$. Afin de savoir si un tel objectif est possible et avec quel risque de perte de rendement, une étude appelée «Ecophyto recherche et développement » a été menée sur la demande du ministère de l'Ecologie et du ministère de l'Agriculture et de la Pêche. Un volet de cette étude porte sur les grandes cultures et analyse l'utilisation des pesticides sur 9 cultures principales dont le colza et le tournesol.

La principale mesure de la pression sanitaire exercée est l'IFT (indicateur de fréquence de traitement) de chaque culture et la surface qu'elle occupe sur le territoire. Cet IFT comptabilise le nombre de doses homologuées appliquées sur un hectare pendant la campagne culturale mais, par contre, ne prend pas en compte les caractéristiques des produits utilisés (toxicité et rémanence par exemple). Malgré cette restriction dans l'information fournie quant aux risques liés à la pression d'utilisation des pesticides, l'étude montre clairement que le colza fait partie des cultures ayant l'IFT le plus élevé, ceci sans doute en raison des nombreux bioagresseurs auxquels il est sensible (phoma, oïdium, sclérotinia ; limaces; grosses altises, charançons du bourgeon de la tige ou des siliques, puceron cendré, méligèthes et bien d'autres insectes à moindre titre ; flore adven- 
tice). Parmi les pesticides, ce sont les insecticides qui ont connu la plus forte augmentation $d^{\prime}$ IFT et ceci semble en outre corrélé avec une augmentation des surfaces cultivées qui sont passées de 693000 ha en 1994 à 1400000 ha en 2006. Pour ce qui est du tournesol, I'IFT concerne surtout les herbicides et il faut noter que les produits sanitaires sont utilisés de manière assez homogène sur le territoire, contrairement au colza qui est très fortement traité dans certaines régions. Dans ce rapport, les incidences de la réduction des produits phytosanitaires sont évaluées selon 3 scénarios majeurs de technique de rupture. Nous n'aborderons pas ici les résultats obtenus selon les niveaux ou sous-niveaux de rupture envisagés ni la manière $d^{\prime} y$ parvenir.

Par contre, compte tenu de ce que nous avons rapporté plus haut sur l'impact direct ou indirect, immédiat ou différé des insecticides, de la rémanence des produits ou de leurs interactions possibles avec les fongicides voire des herbicides, il est illusoire d'étudier tous les risques combinés possibles. La conduite la plus raisonnable à tenir est la réduction de l'ensemble de ces produits dans les limites calculées et acceptées d'une réduction du rendement ou de la valeur économique de la production.

Privilégier la réduction des insecticides, fongicides ou herbicides tout au long de la végétation, en ne les utilisant qu'en cas de besoin avéré, suivre strictement les préconisations d'application, constitue le minimum indispensable à respecter. Pour arriver concrètement à cela, en ce qui concerne le cas particulier de la protection de l'abeille domestique, il faut lever la barrière (pour ne pas dire les conflits) qui existe bien trop souvent entre apiculteurs et agriculteurs. Abeilles et plantes ont depuis toujours une relation mutualiste, pourquoi n'en serait-il pas de même entre deux catégories de l'espèce humaine? Chacun aurait tout à y gagner, c'est la définition même du mutualisme.

\section{RÉFÉRENCES}

1. Alix A, Vergnet C. Risk assessment to honey bees: a scheme developped in France for nonspayed systemic compounds. Pest management Science $2007 ; 63$ : 1069-80.

2. Subirana M. Proposition de protocole d'étude sous tunnel des effets de produits phytopharmaceutiques sur une colonie d'abeilles. CNDA infos $2005 ; 12$ : 7-9.

3. Aupinel $P$, Fortini $D$, Dufour $H$, Tasei $J N$, Michaud B, Odoux JF. Improvement of artificial feeding in a standard in vitro method for rearing Apis mellifera larvae. Bull Insectol 2005 ; 58 : 107-11.

4. Giffard H, Dupont T. L'usage de la phosalone en expérimentation sur abeilles. Phytoma $2001 ; 538: 11-2$.

5. Colin ME, Belzunces LP. Evidence of synergy between prochloraz and deltamethrin : a convenient biological approach. Pesticide Science $1992 ; 36: 115-9$.

6. Kirchner WH. Mad-bee desease? Sublethal effects of imidacloprid "Gaucho" on the behaviour of honey bees. Apidologie $1999 ; 30: 422$.

7. El Hassani A, Dacher M, Gary V, Lambin M, Gauthier M, Armengaud C. Effects of Sublethal Doses of Acetamiprid and Thiamethoxam on the Behavior of the Honeybee (Apis mellifera). Arch Environm Contamination Toxicol 2008; $54: 653-61$.

8. Decourtye A, Tisseur M, Tasei In, PhamDelegue MH. Toxicité et risques liés à l'emploi de pesticides chez les pollinisateurs : le cas de l'abeille domestique. In : Enjeux phytosanitaires pour l'agriculture et l'environnement. RegnaultRoger C ed., Lavoisier éditions TEC \& DOC, 2005 : 225-41.

9. Decourtye A, et al. Comparative sublethal toxicity of nine pesticides on olfactory learning performances of the honeybee Apis mellifera. Arch Environ Contam Toxicol 2005 ; 48 : 242-50.
10. De Boyer Des Roches A, Bournez L, Allier F, Britten V, Vallon J, Jourdan P. Estimation des pertes hivernales de colonies d'abeilles (Apis mellifera) chez les apiculteurs professionnels en 20072008 : premiers résultats. Bulletin technique apicole 2009 ; 145 : 33-46.

11. Cox-Foster DL, et al. A metagenomic survey of microbes in honey bee colony collapse disorder. Science 2007 ; 318 : 283-7.

12. Pierre J. Impacts des OGM sur les abeilles: revue bibliographique. Bulletin technique apicole $2007 ; 34$ : 168-175.

13. Chauzat MP, Carpentier P, Martel AC, et al. Influence of pesticides residues on honey bee (Hymenoptera: Apidae) colony health in France. Environ Entomol 2009 ; 38 : 514-23.

14. Klein A, et al. Importance of pollinators in changing landscape of world crops. In: Proceedings of the Royal Society B 2007 ; 274 : 303-13.

15. Gallai N, Salles JM, Settele J, Vaissière BE. Economic evaluation of the vulnerability of world agriculture confronted with pollinator decline. Ecological Economics $2009 ; 68$ : 810-21.

16. Sabbahi R, De Oliveira D, Marceau J. Influence of honey bee (Hymenoptera: Apidae) density on the production of canola (Cruciferae: Brassicacae). J Econ Entomol 2005 ; 98 : 367-72.

17. Pierre J, Vaissiere B, Picault $H$, Renard M. Relative contribution of insects, wind and passive self-pollination to seed production of winter oilseed rape Brassica napus. (soumis à Crop Science).

18. Kremen C, Williams NM, Thorp RW. Crop pollination from native bees at risk from agricultural intensification. PNAS 2002 ; 99 : 16812-6.

19. Decourtye A, Lecompte P, Pierre J, Chauzat MP, Thiebeau $P$. Introduction de jachères florales en zones de grandes cultures : Atouts pour agriculteurs et apiculteurs. Cah Agr 2007 ; 16 : 213-8.

20. Saddier $M$. Pour une filière apicole durable. Les abeilles et les pollinisateurs sauvages.www. agriculture.gouv.fr/./rapports/./Rapport_SADDIER2008 